\section{RMD Open}

Rheumatic \&

Musculoskeletal Diseases

\title{
Psoriasis and the risk of foot and
} ankle tendinopathy or enthesopathy in the absence of psoriatic arthritis: a population-based study

\author{
Ryan T Lewinson, ${ }^{1,2}$ Isabelle A Vallerand, ${ }^{1,3}$ Laurie M Parsons, ${ }^{4}$ \\ Jeremy M LaMothe, ${ }^{5}$ Alexandra D Frolkis, ${ }^{4}$ Mark W Lowerison, ${ }^{3}$ Gilaad G Kaplan, ${ }^{4}$ \\ Scott B Patten, ${ }^{3,6}$ Cheryl Barnabe ${ }^{3,4}$
}

To cite: Lewinson RT, Vallerand IA, Parsons LM, et al. Psoriasis and the risk of foot and ankle tendinopathy or enthesopathy in the absence of psoriatic arthritis: a populationbased study. RMD Open 2018:4:e000668. doi:10.1136/ rmdopen-2018-000668

- Prepublication history for this paper is available online. To view these files please visit the journal online (http://dx.doi. org/10.1136/rmdopen-2018000668).

Received 14 February 2018 Revised 2 April 2018

Accepted 1 May 2018
Check for updates

For numbered affiliations see end of article.

Correspondence to Dr Cheryl Barnabe; ccbarnab@ucalgary.ca

\section{ABSTRACT}

Objectives Imaging studies in patients with cutaneous psoriasis have demonstrated asymptomatic bone and tendon changes, commonly of the foot and ankle. We sought to determine if patients with cutaneous psoriasis have an increased risk of clinically significant foot and ankle tendinopathy or enthesopathy compared with the general population.

Methods Patients with cutaneous psoriasis and a general population cohort were identified in The Health Improvement Network, a general practice medical records database from the UK. All patients with psoriatic arthritis were excluded. Cox proportional-hazards models $(\alpha=0.05)$ estimated the HR for development of foot and ankle tendinopathy or enthesopathy among patients with psoriasis, with adjustment for numerous covariates.

Results In total, 78630 patients with cutaneous psoriasis and 5983338 persons from the general population were identified. In an unadjusted model, patients with cutaneous psoriasis had a $25 \%$ increased risk of developing foot and ankle tendinopathy or enthesopathy compared with the general population (HR $1.25,95 \% \mathrm{Cl} 1.20$ to 1.30 , $\mathrm{p}<0.0001)$. The HR remained unchanged and statistically significant after adjusting for covariates, and in sensitivity analyses.

Conclusions These data suggest that patients with psoriasis can have foot and ankle tendinopathy or enthesopathy without having psoriatic arthritis, presenting a diagnostic challenge to physicians. Further research is needed to elucidate mechanisms contributing to this increased risk.

\section{INTRODUCTION}

Psoriasis is an inflammatory skin disease characterised by pruritic, erythematous, scaling plaques, papules and occasionally pustules. ${ }^{2}$ While originally thought to be a disease limited to the skin, recent evidence suggests that systemic consequences occur in psoriasis, such as increased risk of myocardial infarction, ${ }^{3}$ chronic kidney disease, ${ }^{4}$ cancer $^{5}$ and depression. ${ }^{6}$

\section{Key messages}

What is already known about this subject?

- Imaging studies suggest that many patients with psoriasis have asymptomatic foot and ankle bone and tendon lesions.

What does this study add?

- Patients with cutaneous psoriasis had a $25 \%$ increased risk of developing tendinopathy or enthesopathy of the foot and ankle.

How might this impact on clinical practice?

- Patients with psoriasis may present with foot and ankle tendinopathy or enthesopathy without necessarily having psoriatic arthritis.

From a musculoskeletal perspective, $8 \%-30 \%$ of patients with psoriasis develop psoriatic arthritis, ${ }^{78}$ a chronic inflammatory arthropathy. ${ }^{8}$ While joint involvement is the main distinguishing feature between psoriatic arthritis and psoriasis, a recognised extra-articular feature of psoriatic arthritis is inflammation of tendons, usually at their insertions or entheses, termed tendinopathy and enthesopathy respectively. ${ }^{89}$ Most commonly, this manifests at the plantar fascia and Achilles tendons, ${ }^{9}$ causing significant foot and ankle pain and immobility.

Tendon and entheseal involvement is typically considered a feature exclusive to psoriatic arthritis rather than psoriasis. However, recent studies using high-resolution peripheral quantitative CT scans have shown that psoriasis patients without arthritis had significant enthesophyte formation, signalling enthesopathy, compared with healthy controls. $^{10}$ Moreover, in an MRI study of patients with psoriasis, it was found that $57 \%$ had evidence of asymptomatic Achilles tendinopathy. ${ }^{11}$ On ultrasound imaging, patients 


\begin{tabular}{|c|c|}
\hline N217400 & Achilles tendinitis \\
\hline N217500 & Tibialis anterior tendinitis \\
\hline N217600 & Tibialis posterior tendinitis \\
\hline N217900 & Plantar fasciitis \\
\hline N217.00 & Enthesopathy of the ankle and tarsus \\
\hline N217z00 & Ankle or tarsus enthesopathy NOS \\
\hline N217000 & Enthesopathy of the ankle unspecified \\
\hline N217100 & Enthesopathy of the tarsus unspecified \\
\hline NyuAD00[X] & Other enthesopathy of foot \\
\hline N217.11 & Tarsus enthesopathy \\
\hline
\end{tabular}

Consensus on which codes to use was established by a

rheumatologist and orthopaedic surgeon.

with spondyloarthropathy had more frequent enthesopathy in the absence of clinical symptoms compared with healthy controls. ${ }^{12}$ Thus, it appears that patients with psoriasis may develop pathology consistent with tendon dysfunction, but since these studies were relatively small and without clinical follow-up, it remains unknown whether patients with psoriasis actually experience clinically significant tendon and entheseal disorders at a greater rate than the general population.

In this study, we used The Health Improvement Network (THIN), a general practice medical records database in the UK, to test the hypothesis that psoriasis patients without psoriatic arthritis are at increased risk of developing clinically significant foot and ankle tendinopathy or enthesopathy compared with the general population, thereby identifying a potential extracutaneous manifestation. We focused exclusively on the foot and ankle (a) as these are the most common sites observed for tendon dysfunction in patients with psoriatic arthritis (ie, Achilles tendon and plantar fascia), ${ }^{9}$ (b) as previous research has shown imaging evidence of disease at these sites in patients with psoriasis ${ }^{11}$ and (c) to explore the concept of a 'deep Koebner' phenomenon, with selection of a site with high mechanical loading offering pathophysiological insight.

\section{METHODS}

\section{Data source}

THIN is an electronic database that contains general practice medical records from the UK with up to 25 years of follow-up. ${ }^{13}$ Additionally, data from specialists are provided to general practitioners and subsequently recorded in THIN. Given the longitudinal follow-up and detailed medical history data, THIN is ideal for studying potentially low-incidence outcomes such as tendinopathy and enthesopathy among a cohort with psoriasis.

\section{Study population and exposure}

THIN was used to identify individuals between the ages of 10-90 years diagnosed with psoriasis (exposure cohort) and those without psoriasis (referent cohort). Diagnosis of psoriasis was based on the presence of one or more validated Read diagnostic codes (linked to International Classification of Diseases codes) for psoriasis. ${ }^{14}$ Any patient with a psoriasis code within 1 year of registering in THIN was excluded from this study, to ensure that all cases of psoriasis were incident, as done previously. ${ }^{15}$ Additionally, to ensure focus on cutaneous psoriasis patients without arthritis, any patient with a validated Read code for psoriatic arthritis at any time in their record was excluded from this study. ${ }^{16}$ Finally, any patients with a Read code for foot and ankle tendinopathy or enthesopathy (main outcome) prior to psoriasis diagnosis were excluded.

The referent cohort (general population) also had a 1-year washout period, and any patient who had a prevalent code for foot and ankle tendinopathy or enthesopathy within this period was excluded from analysis.

\section{Outcomes}

All patients were followed until the earliest of (a) development of foot and ankle tendinopathy or enthesopathy (main outcome), (b) transfer out of practice, (c) death or (d) end of data collection period. Patients who did not have a record of tendinopathy or enthesopathy during this follow-up period were censored. Development of foot and ankle tendinopathy or enthesopathy was defined based on the presence of any Read code relating to tendinopathy or enthesopathy of the foot and ankle. Read codes that were determined to relate to foot and ankle tendinopathy and enthesopathy were identified as follows: first, all Read codes relating to tendinopathy or enthesopathy were identified. This list was then reviewed independently by a rheumatologist and orthopaedic surgeon, where consensus was established on which codes would properly capture tendinopathy or enthesopathy specific to the foot and ankle. While enthesitis is the more common outcome for patients with psoriatic arthritis, tendinopathy was included to broaden our definition since this also occurs in psoriatic arthritis. ${ }^{17}$ Additionally, since clinically defining tendon injury as tendinopathy versus enthesopathy is dependent only on the point along the tendon at which the injury occurs, we considered both terms in our analysis in hopes of improved sensitivity, given not all patients with tendon pain may have imaging to confirm where along the tendon the injury was to classify them as enthesopathy or tendinopathy. Read codes used in our analysis for foot and ankle tendinopathy or enthesopathy can be found in table 1.

\section{Covariates}

Age, sex, obesity status (body mass index $\geq 30 \mathrm{~kg} / \mathrm{m}^{2}$ ), alcohol use (yes or no), smoking status (current, past or never) and socioeconomic status (based on Townsend deprivation index) were determined for all patients in both the psoriasis and referent cohorts. These were taken in closest proximity to the date of diagnosis in the 
Table 2 Baseline characteristics of patients with psoriasis and the general population

\begin{tabular}{|c|c|c|c|}
\hline Variable & $\begin{array}{l}\text { Psoriasis } \\
(n=78630)\end{array}$ & $\begin{array}{l}\text { General population } \\
(n=5983338)\end{array}$ & $P$ values \\
\hline Age (years) & & & $<0.0001$ \\
\hline 10-39years & $38585(49.1 \%)$ & 3446247 (57.6\%) & \\
\hline 40-90 years & 40045 (50.9\%) & $2537091(42.4 \%)$ & \\
\hline Sex & & & 0.529 \\
\hline Females & $40749(51.8 \%)$ & 3094037 (51.7\%) & \\
\hline Obesity status $\left(\mathrm{kg} / \mathrm{m}^{2}\right)$ & & & $<0.0001$ \\
\hline $\mathrm{BMl}<30$ & $28892(36.7 \%)$ & 2502902 (41.8\%) & \\
\hline $\mathrm{BMI} \geq 30$ & $6497(8.3 \%)$ & $433773(7.2 \%)$ & \\
\hline Missing & $43241(55.0 \%)$ & $3046663(50.9 \%)$ & \\
\hline Smoking status & & & $<0.0001$ \\
\hline Current & $19455(24.7 \%)$ & $1204834(20.1 \%)$ & \\
\hline Ex-smoker & $7384(9.4 \%)$ & 540987 (9.0\%) & \\
\hline Never & $34876(44.4 \%)$ & $2996546(50.1 \%)$ & \\
\hline Missing & $16915(21.5 \%)$ & $1240971(20.7 \%)$ & \\
\hline Alcohol use & & & $<0.0001$ \\
\hline User & $31598(40.2 \%)$ & $2634769(44.0 \%)$ & \\
\hline Non-user & $14949(19.0 \%)$ & $1033560(17.3 \%)$ & \\
\hline Missing & $32083(40.8 \%)$ & 2315009 (38.7\%) & \\
\hline Charlson Comorbidity Index* & & & $<0.0001$ \\
\hline 0 & 62609 (79.6\%) & 4817790 (80.5\%) & \\
\hline 1 & $11258(14.3 \%)$ & $754495(12.6 \%)$ & \\
\hline 2 & $2238(2.8 \%)$ & $165226(2.8 \%)$ & \\
\hline 3 & $813(1.0 \%)$ & $62700(1.0 \%)$ & \\
\hline$\geq 4$ & $1712(2.2 \%)$ & $183127(3.1 \%)$ & \\
\hline Townsend Deprivation Index† & & & $<0.0001$ \\
\hline 1 & $18438(23.4 \%)$ & $1289151(21.5 \%)$ & \\
\hline 2 & $16178(20.6 \%)$ & $1147462(19.2 \%)$ & \\
\hline 3 & 15616 (19.9\%) & 1157934 (19.4\%) & \\
\hline 4 & $14224(18.1 \%)$ & 1103255 (18.4\%) & \\
\hline 5 & $10144(12.9 \%)$ & $802761(13.4 \%)$ & \\
\hline Missing & $4030(5.1 \%)$ & $482775(8.1 \%)$ & \\
\hline Systemic psoriasis therapy & & & $<0.0001$ \\
\hline User & $3191(4.1 \%)$ & $50172(0.8 \%)$ & \\
\hline Systemic steroid use & & & $<0.0001$ \\
\hline User & $19738(25.1 \%)$ & $748707(12.5 \%)$ & \\
\hline Fluoroquinolone use & & & $<0.0001$ \\
\hline User & 12445 (15.8\%) & 480857 (8.0\%) & \\
\hline
\end{tabular}

Values show the number (\%) of patients with a given characteristic. Moderate-severe psoriasis defined based on therapy use (see Methods section).

${ }^{*}$ Higher=more comorbidities.

†Higher=more socially deprived.

psoriasis cohort and closest to the start date in THIN for the referent cohort, as done previously. ${ }^{15}$ We also assessed comorbidity burden using Read codes to compute the Charlson comorbidity index within the first three years of the study start date. ${ }^{15}$
As patients with moderate-severe psoriasis are likely to receive systemic therapy which may mask or treat tendinopathy or enthesopathy, we also recorded use of systemic therapy as a covariate defined based on the presence of any of the following codes, as done previously ${ }^{315}$ : 
acitretin, azathioprine, cyclosporine, etretinate, hydroxycarbamide, methotrexate, mycophenolic acid and phototherapy with or without psoralen. Biologics were not considered as they are poorly recorded in THIN ${ }^{15}{ }^{16}$; however, since UK treatment guidelines indicate patients with psoriasis should be started on a disease-modifying antirheumatic drugs prior to any biologic, ${ }^{18}$ likely these patients would still be captured within our systemic therapy covariate.

Lastly, we included use of systemic steroid medications and use of fluoroquinolones at any point during the study period as covariates as these medications are known to predispose to tendon injury. ${ }^{19-21}$

\section{Statistical analysis and data presentation}

This manuscript was prepared in accordance with the Strengthening the Reporting of Observational Studies in Epidemiology statement. ${ }^{22}$ Analyses were performed using STATA/MP V.13.1 $(\alpha=0.05)$. Baseline data were compared between the psoriasis and referent cohort using $\chi^{2}$ tests. Cox proportional-hazards models were used to evaluate the risk of foot and ankle tendinopathy or enthesopathy among psoriasis patients compared with the referent cohort, where risk was estimated as an HR with 95\% CIs. An omnibus likelihood ratio test was used to assess for statistical interactions between all covariates found to have a significant difference between groups at baseline,$^{15}$ and no interactions were identified $(p=0.189)$. A backward elimination procedure was then used to evaluate each covariate for possible confounding starting with a fully adjusted model. Ultimately, no variables were found to induce an important change to the HR (ie, $>10 \%$ ) to suggest confounding. ${ }^{15}$ Data were then presented in both an unadjusted and fully adjusted model. The proportional-hazards assumption was evaluated by testing the Schoenfeld residuals and constructing a $\log -\log$ plot, and no evidence against this assumption was found.

To further ensure that the psoriasis cohort did not contain individuals likely to develop psoriatic arthritis, a sensitivity analysis was performed that excluded patients who had a code for uveitis, which is known to be a substantial risk factor for psoriatic arthritis in patients with psoriasis. ${ }^{23}$ In addition, all patients with codes for inflammatory bowel disease or ankylosing spondylitis were removed from consideration to ensure enthesopathy or tendinopathy were not due to these conditions. ${ }^{24}{ }^{25}$ To confirm only incident psoriasis cases were included, a sensitivity analysis was performed where patients had to be registered in THIN for at least 5 years before psoriasis diagnosis. Finally, to ensure adequate time was available to develop the outcome of interest, an additional sensitivity analysis was performed that restricted to only patients with at least 5 years of follow-up from their start date in THIN.

\section{Data availability}

The data that support the findings of this study are available from IMS Health (UK) but restrictions apply to the
Table 3 HRs for the risk of foot and ankle tendinopathy and enthesopathy

\begin{tabular}{lll}
\hline Model & HR $(95 \%$ Cl) & P values \\
\hline \begin{tabular}{l} 
Unadjusted model \\
\multicolumn{1}{l}{ Psoriasis }
\end{tabular} & $1.25(1.20$ to 1.30$)$ & $<0.0001$ \\
\hline $\begin{array}{l}\text { Multivariable adjusted model } \\
\text { Psoriasis }\end{array}$ & $1.25(1.17$ to 1.33$)$ & $<0.0001$ \\
\hline Age & $1.44(1.42$ to 1.47$)$ & $<0.0001$ \\
\hline $\begin{array}{l}\text { Obesity status } \\
\text { Smoking status }\end{array}$ & $2.08(2.04$ to 2.12$)$ & $<0.0001$ \\
\hline $\begin{array}{l}\text { Alcohol use } \\
\text { Charlson Comorbidity }\end{array}$ & $0.92(0.91$ to 0.93$)$ & $<0.0001$ \\
\hline $\begin{array}{l}\text { Index } \\
\text { Townsend Deprivation } \\
\text { Index }\end{array}$ & $1.14(1.12$ to 1.16$)$ & $<0.0001$ \\
\hline $\begin{array}{l}\text { Systemic psoriasis } \\
\text { therapy }\end{array}$ & $0.61(0.16$ to 1.21$)$ & $<0.0001$ \\
\hline $\begin{array}{l}\text { Systemic steroid use } \\
\text { Fluoroquinolone use }\end{array}$ & $0.88(0.86$ to 0.90$)$ & $<0.0001$ \\
\hline
\end{tabular}

Cox proportional-hazards models were used to estimate the Hrs of developing foot and ankle tendinopathy or enthesopathy based on whether patients had psoriasis or not (ie, psoriasis vs general population). As can be seen from these models, psoriasis significantly increases the risk of developing foot and ankle tendinopathy or enthesopathy when using unadjusted models as well as models accounting for numerous covariates.

availability of these data, which were used under licence for the current study, and so are not publicly available. Data are however available from the authors on reasonable request and with permission of IMS Health.

\section{RESULTS}

Using THIN, and after ensuring all patients with a code for psoriatic arthritis were removed from consideration, we identified 78630 patients with cutaneous psoriasis, and a referent cohort comprising 5983338 patients (figure 1). At baseline (table 2), the following covariates were found in a greater proportion among the psoriasis cohort: age $>40$ years, presence of obesity, current smoking, comorbidities, systemic therapy use, systemic steroid use and fluoroquinolone use (all $\mathrm{p}<0.0001$ ). Conversely, the psoriasis cohort had a lower proportion of individuals who were socioeconomically deprived or alcohol users. Sex distributions were similar between the psoriasis and referent cohorts $(\mathrm{p}=0.529)$.

Follow-up times were up to 26 years, with a median follow-up of 5.1 years (IQR 6.9 years) in the psoriasis cohort and 5.6 years (IQR 7.0 years) in the general population. In total, 2631 patients with psoriasis (3.3\%) developed foot and ankle tendinopathy or enthesopathy while 147919 patients $(2.5 \%)$ developed foot and ankle tendinopathy or enthesopathy in the general population. The median time to development of foot and ankle tendinopathy or enthesopathy from psoriasis diagnosis was 


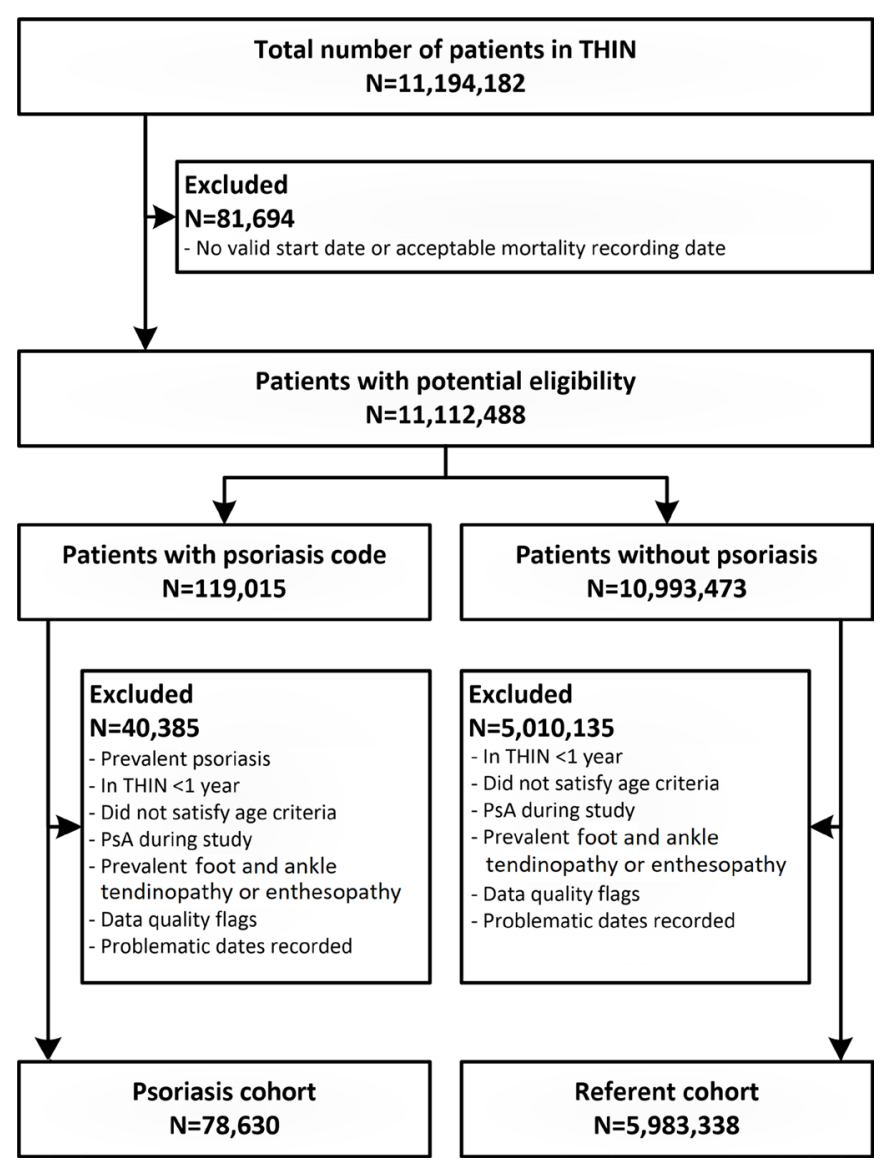

Figure 1 Flow chart showing selection of patients in the psoriasis and general population referent cohort. PsA, psoriatic arthritis; THIN, The Health Improvement Network.

4.4 years (IQR 5.8 years). Overall, the median age at diagnosis of foot and ankle tendinopathy or enthesopathy was 53.2 years (IQR 21.6 years).

An unadjusted cox proportional-hazards model revealed that patients with psoriasis were at a $25 \%$

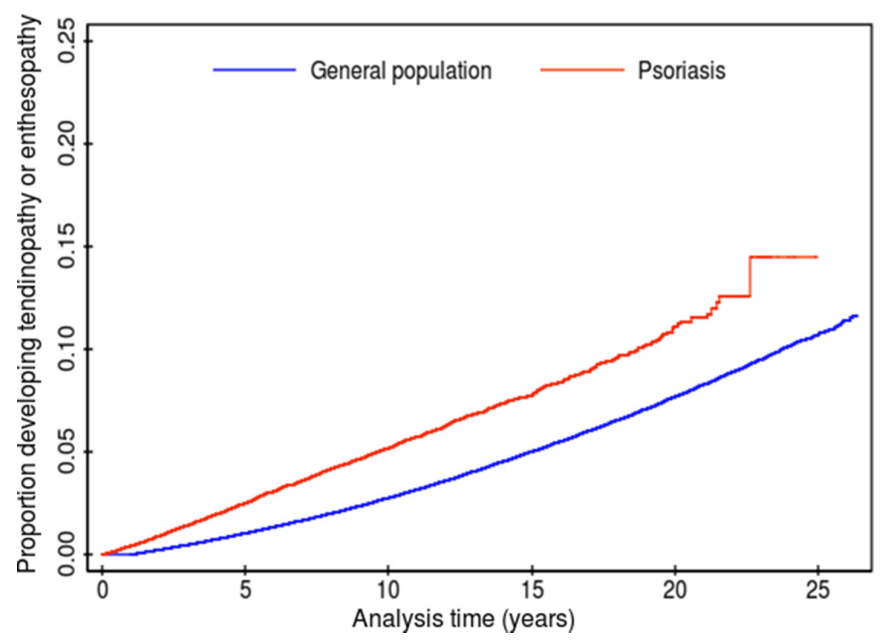

Figure 2 Kaplan-Meier failure curve for development of foot and ankle tendinopathy or enthesopathy. Patients with psoriasis (orange) were at a $25 \%$ increased risk of foot and ankle tendinopathy or enthesopathy compared with the general population (blue). increased risk of developing foot and ankle tendinopathy or enthesopathy compared with the general population (HR $1.25,95 \%$ CI 1.20 to $1.30, \mathrm{p}<0.0001$ ). All covariates that were significantly different between groups at baseline were included in a fully adjusted model, and patients with psoriasis remained at significantly increased risk of foot and ankle tendinopathy or enthesopathy (HR 1.25, $95 \%$ CI 1.17 to $1.33, \mathrm{p}<0.0001)$. These models are shown in table 3. Kaplan-Meier failure curves for development of foot and ankle tendinopathy or enthesopathy can be seen in figure 2.

To further ensure we excluded patients with a predisposition to tendinopathy or enthesopathy through other autoimmune processes, ${ }^{23}$ we performed sensitivity analyses to exclude 42215 patients with uveitis, inflammatory bowel disease or ankylosing spondylitis. This resulted in 77326 patients with psoriasis and 5942427 patients from the general population analysed. Here, in the fully adjusted model, psoriasis remained a significant risk factor for development of foot and ankle tendinopathy or enthesopathy (HR 1.25, 95\% CI 1.17 to 1.34 , $\mathrm{p}<0.0001)$. Similarly, results remained unchanged when restricting to only those patients with at least 5 years in THIN before psoriasis diagnosis (HR 1.30, 95\% CI 1.18 to $1.43, \mathrm{p}<0.0001$ ) or when restricting to only those patients who had at least 5 years of follow-up in THIN (HR 1.25, 1.16 to $1.34, \mathrm{p}<0.0001)$.

\section{DISCUSSION}

The absolute risk of foot and ankle tendinopathy or enthesopathy was low in both the psoriasis and referent cohorts. Thus, clinically it is important to acknowledge that foot and ankle tendinopathy or enthesopathy is not experienced by a large proportion of patients with psoriasis; however, our finding of a $25 \%$ increased risk of foot and ankle tendinopathy or enthesopathy among those with psoriasis may provide important information on the underlying pathophysiology of psoriasis, and add to the growing body of evidence suggestive of systemic pathophysiology in the development and course of psoriasis.

Previous research has shown that patients with psoriasis have imaging findings consistent with tendon dysfunction more frequently than healthy controls. ${ }^{11}$ Similarly, subclinical enthesitis has been documented in patients with psoriatic arthritis. ${ }^{26}$ Although some overlap exists in genetic architecture between psoriasis and psoriatic arthritis, each condition and specific disease phenotypes have unique genetic signatures, perhaps accounting for why not all patients with psoriasis develop psoriatic arthritis, or why some patients develop arthritis before psoriasis. ${ }^{27} 28$ Our study adds to this complex relationship by identifying that patients with psoriasis are at $25 \%$ increased risk of being diagnosed with foot and ankle tendinopathy or enthesopathy by a physician compared with the general population. Clinically, this indicates that foot and ankle tendinopathy or enthesopathy is associated with psoriasis and not only psoriatic arthritis. Just 
as some patients with psoriasis show susceptibility to development of articular or nail symptoms, ${ }^{28}$ it might also be possible that some patients with psoriasis are at higher susceptibility to tendon or enthesis symptoms, but without joint symptoms to diagnose them as having psoriatic arthritis. Since we excluded all patients with psoriatic arthritis codes in our study, and further excluded those with uveitis, inflammatory bowel disease and ankylosing spondylitis, this poses a clinical challenge to physicians as patients may experience foot and ankle tendinopathy or enthesopathy without necessarily having psoriatic arthritis, leading to uncertainty on the diagnosis or the need for rheumatology consultation.

The diagnosis of psoriatic arthritis is largely clinical since there are no specific genetic, tissue biopsy or serological markers. While many different clinical classification criteria exist for psoriatic arthritis, it should be emphasised that these are simply a means to classify disease phenotypes rather than diagnostic criteria for psoriatic arthritis. On the assumption of patients exclusively having psoriasis without arthritis but with findings of tendinopathy or enthesopathy, many clinical criteria would not classify these patients as having psoriatic arthritis. While it remains possible that a subset of the patients considered in this study had additional features that would classify them as psoriatic arthritis by some criteria, these patients would be expected to evolve over time and eventually be captured and recoded as having psoriatic arthritis in THIN, which we excluded from analysis. Moreover, by exclusion of patients with codes for uveitis, which has been documented as a strong risk factor for future development of psoriatic arthritis, ${ }^{23}$ by eliminating all patients with inflammatory bowel disease or ankylosing spondylitis from consideration and by restricting to only patients with a long follow-up time in sensitivity analyses, the possibility that remaining patients would subsequently develop joint involvement and psoriatic arthritis is low. Thus, our results more likely represent foot and ankle tendinopathy or enthesopathy in the setting of cutaneous psoriasis rather than psoriatic arthritis. The alternative to this possibility, depending on the classification system chosen, is that these patients simply represent unrecognised cases of psoriatic arthritis; however, this is perhaps less likely based on the sensitivity analyses performed, and on the basis that any misclassification bias would be expected to occur non-differentially between the psoriasis and referent cohorts.

Given that the Achilles tendon and plantar fascia are typically sites of repetitive mechanical loading, the possibility of a biomechanical aetiology in foot and ankle tendinopathy or enthesopathy is strong. It is well documented that repetitive mechanical stress in the Achilles tendon and plantar fascia can alter gene expression, induce local inflammation and tendon degradation, ${ }^{29-31}$ giving support to the proposed concept of a 'deep Koebner phenomenon' in psoriatic disease. ${ }^{10}{ }^{32}$ Indeed, it has recently been proposed that mechanical stress may be an important factor contributing to the onset of inflammation and development of spondyloarthritis. ${ }^{33}$ Moreover, tendon degradation may persist following chronic under loading, ${ }^{29}$ and interestingly one study involving patients with psoriatic arthritis with active enthesitis did demonstrate reduced Achilles tendon force during gait. ${ }^{34}$ While it has been suggested that the biomechanical environment of the Achilles tendon and plantar fascia may be dependent on underlying muscle physiology, ${ }^{35-37}$ musculoskeletal anatomy, ${ }^{35} 38$ obesity, ${ }^{39}$ gait abnormalities ${ }^{38}$ and even footwear, ${ }^{40}{ }^{41}$ none of these factors have been assessed in the context of foot and ankle pathology in patients with psoriasis. Therefore, additional research in this area is warranted to understand the potential mechanisms by which patients with psoriasis develop foot and ankle tendinopathy or enthesopathy.

This study has a number of strengths and limitations to consider. First, our use of a large, nationally representative database allowed us to study very large sample sizes over a long period of time. While this gave substantial statistical power, this also led to small differences being statistically significant, and these should be interpreted from a frame of clinical relevance. Second, by using a medical database there is also a risk of misclassification of cases. However, previous work has validated codes for psoriasis and psoriatic arthritis, ${ }^{14} 16$ and our results remained robust in sensitivity analyses, so this risk is expected to be small. Nonetheless, it is possible that some patients had psoriatic arthritis prior to beginning in THIN and were subsequently not captured and removed from this study. Lastly, while this study shows an association between psoriasis and foot and ankle tendinopathy or enthesopathy, it was not possible to include imaging findings, genetics or serum inflammatory markers, which may provide further information.

In summary, this study suggests that cutaneous psoriasis without joint involvement increases the risk of developing foot and ankle tendinopathy or enthesopathy by approximately $25 \%$. While the absolute risk of developing foot and ankle tendinopathy or enthesopathy among patients with psoriasis was relatively low, the increased risk relative to the general population is important in considering the potential systemic nature of psoriasis. Future research should explore the mechanisms by which patients with psoriasis are at increased risk of foot and ankle tendinopathy or enthesopathy. Clinically, physicians should be aware that patients with psoriasis may present with foot and ankle tendinopathy or enthesopathy without necessarily having psoriatic arthritis.

\section{Author affiliations}

${ }^{1}$ Leaders in Medicine Program, Cumming School of Medicine, University of Calgary, Calgary, Alberta, Canada

${ }^{2}$ Biomedical Engineering Program, Schulich School of Engineering, University of Calgary, Calgary, Alberta, Canada

${ }^{3}$ Department of Community Health Sciences, Cumming School of Medicine, University of Calgary, Calgary, Alberta, Canada

${ }^{4}$ Department of Medicine, Cumming School of Medicine, University of Calgary, Calgary, Alberta, Canada 
${ }^{5}$ Department of Surgery, Cumming School of Medicine, University of Calgary, Calgary, Alberta, Canada

${ }^{6}$ Department of Psychiatry, Cumming School of Medicine, University of Calgary, Calgary, Alberta, Canada

Correction notice This article has been corrected since it first published. Figures 1 and 2 have been transposed so that they are with the correct figure legend.

Contributors RTL, IAV and CB developed the study topic. LMP, JML and CB contributed expert advice on psoriasis, psoriatic arthritis and tendinopathy/ enthesopathy. RTL, LMP, JML and CB reviewed Read codes corresponding to the present study. IAV, ADF and MWL contributed to data systems development and extraction. GGK, SBP and CB provided methodological expertise. Statistica analyses were performed by IAV with critical review by RTL, GGK, SBP and CB. RTL drafted the manuscript, tables and figures, with all authors providing critical review. All authors approved the final version of this manuscript.

Funding This study was funded by a studentship from the Canadian Association of Psoriasis Patients and the Canadian Institutes of Health Research.

Competing interests None declared.

Patient consent Not required.

Ethics approval Approved by the University of Calgary Conjoint Health Research Ethics Board and the UK IMS Health Scientific Review Committee.

Provenance and peer review Not commissioned; externally peer reviewed.

Open Access This is an Open Access article distributed in accordance with the Creative Commons Attribution Non Commercial (CC BY-NC 4.0) license, which permits others to distribute, remix, adapt, build upon this work non-commercially, and license their derivative works on different terms, provided the original work is properly cited and the use is non-commercial. See: http://creativecommons.org/ licenses/by-nc/4.0/

(C) Article author(s) (or their employer(s) unless otherwise stated in the text of the article) 2018. All rights reserved. No commercial use is permitted unless otherwise expressly granted.

\section{REFERENCES}

1. Nestle FO, Kaplan DH, Barker J. Psoriasis. N Engl J Med 2009;361:496-509.

2. Boehncke WH, Schön MP. Psoriasis. Lancet 2015;386:983-94

3. Gelfand JM, Neimann AL, Shin DB, et al. Risk of myocardial infarction in patients with psoriasis. JAMA 2006;296:1735-41.

4. Wan J, Wang S, Haynes K, et al. Risk of moderate to advanced kidney disease in patients with psoriasis: population based cohort study. BMJ 2013;347:f5961

5. Chiesa Fuxench ZC, Shin DB, Ogdie Beatty A, et al. The risk of cancer in patients with psoriasis: a population-based cohort study in the health improvement network. JAMA Dermatol 2016;152:282-90.

6. Kurd SK, Troxel AB, Crits-Christoph P, et al. The risk of depression, anxiety, and suicidality in patients with psoriasis: a population-based cohort study. Arch Dermatol 2010;146:891-5.

7. Ogdie A, Langan S, Love T, et al. Prevalence and treatment patterns of psoriatic arthritis in the UK. Rheumatology 2013;52:568-75.

8. Ritchlin CT, Colbert RA, Gladman DD, et al. Psoriatic arthritis. N Engl J Med 2017:376:957-70.

9. Polachek A, Li S, Chandran V, et al. Clinical enthesitis in a prospective longitudinal psoriatic arthritis cohort: incidence, prevalence, characteristics and outcome. Arthritis Care Res 2016.

10. Simon D, Faustini F, Kleyer A, et al. Analysis of periarticular bone changes in patients with cutaneous psoriasis without associated psoriatic arthritis. Ann Rheum Dis 2016;75:660-6.

11. Erdem CZ, Tekin NS, Sarikaya S, et al. MR imaging features of foot involvement in patients with psoriasis. Eur J Radiol 2008;67:521-5.

12. Ruta S, Gutierrez M, Pena C, et al. Prevalence of subclinical enthesopathy in patients with spondyloarthropathy: an ultrasound study. J Clin Rheumatol 2011;17:18-22.

13. IMS Health U. Statistics. 2016 http://www.csdmruk.imshealth.com/ our-data/statistics.shtml.

14. Seminara NM, Abuabara K, Shin DB, et al. Validity of The Health Improvement Network (THIN) for the study of psoriasis. $\mathrm{Br} \mathrm{J}$ Dermatol 2011;164:602-9.

15. Lewinson RT, Vallerand IA, Lowerison MW, et al. Depression is associated with an increased risk of psoriatic arthritis among patients with psoriasis: a population-based study. J Invest Dermatol 2017;137:828-35
16. Ogdie A, Alehashemi S, Love TJ, et al. Validity of psoriatic arthritis and capture of disease modifying antirheumatic drugs in the health improvement network. Pharmacoepidemiol Drug Saf 2014;23:918-22.

17. Gutierrez M, Filippucci E, De Angelis R, et al. A sonographic spectrum of psoriatic arthritis: "the five targets". Clin Rheumatol 2010;29:133-42.

18. Excellence NIfHaC. Psoriasis: assessment and management, NICE Guideline [CG153]. 2012. 2012. updated 2017.

19. Stephenson AL, Wu W, Cortes D, et al. Tendon injury and fluoroquinolone use: a systematic review. Drug Saf 2013;36:709-21.

20. van der Linden PD, Sturkenboom MC, Herings RM, et al. Increased risk of achilles tendon rupture with quinolone antibacterial use, especially in elderly patients taking oral corticosteroids. Arch Intern Med 2003;163:1801-7.

21. Newnham DM, Douglas JG, Legge JS, et al. Achilles tendon rupture: an underrated complication of corticosteroid treatment. Thorax 1991;46:853-4.

22. von Elm E, Altman DG, Egger M, et al. The Strengthening the Reporting of Observational Studies in Epidemiology (STROBE) statement: guidelines for reporting observational studies. Lancet 2007;370:1453-7.

23. Eder L, Haddad A, Rosen CF, et al. The incidence and risk factors for psoriatic arthritis in patients with psoriasis: a prospective cohort study. Arthritis Rheumatol 2016;68:915-23.

24. Dubreuil M, Peloquin $\mathrm{C}$, Zhang $\mathrm{Y}$, et al. Validity of ankylosing spondylitis diagnoses in The Health Improvement Network. Pharmacoepidemiol Drug Saf 2016;25:399-404.

25. Frolkis $A D$, de Bruyn J, Jette $\mathrm{N}$, et al. The association of smoking and surgery in inflammatory bowel disease is modified by age at diagnosis. Clin Trans/ Gastroenterol 2016;7:e165.

26. Weckbach S, Schewe S, Michaely HJ, et al. Whole-body MR imaging in psoriatic arthritis: additional value for therapeutic decision making. Eur J Radiol 2011;77:149-55.

27. Stuart PE, Nair RP, Tsoi LC, et al. Genome-wide association analysis of psoriatic arthritis and cutaneous psoriasis reveals differences in their genetic architecture. Am J Hum Genet 2015;97:816-36.

28. Haroon M, Winchester R, Giles JT, et al. Certain class I HLA alleles and haplotypes implicated in susceptibility play a role in determining specific features of the psoriatic arthritis phenotype. Ann Rheum Dis 2016;75:155-62.

29. Galloway MT, Lalley AL, Shearn JT. The role of mechanical loading in tendon development, maintenance, injury, and repair. J Bone Joint Surg Am 2013;95:1620-8.

30. Rees JD, Stride M, Scott A. Tendons--time to revisit inflammation. $\mathrm{Br}$ J Sports Med 2014;48:1553-7.

31. Mendias CL, Gumucio JP, Bakhurin Kl, et al. Physiological loading of tendons induces scleraxis expression in epitenon fibroblasts. $J$ Orthop Res 2012;30:606-12.

32. Yamamoto T. Psoriatic arthritis: from a dermatological perspective. Eur J Dermatol 2011;21:660-6.

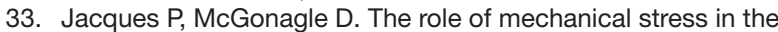
pathogenesis of spondyloarthritis and how to combat it. Best Pract Res Clin Rheumatol 2014;28:703-10.

34. Woodburn J, Hyslop E, Barn R, et al. Achilles tendon biomechanics in psoriatic arthritis patients with ultrasound proven enthesitis. Scand J Rheumatol 2013;42:299-302.

35. Lersch C, Grötsch A, Segesser B, et al. Influence of calcaneus angle and muscle forces on strain distribution in the human Achilles tendon. Clin Biomech 2012;27:955-61.

36. Mahieu NN, Witvrouw E, Stevens V, et al. Intrinsic risk factors for the development of achilles tendon overuse injury: a prospective study. Am J Sports Med 2006;34:226-35.

37. Chen YN, Chang CW, Li CT, et al. Finite element analysis of plantar fascia during walking: a quasi-static simulation. Foot Ankle Int 2015;36:90-7.

38. Pohl MB, Hamill J, Davis IS. Biomechanical and anatomic factors associated with a history of plantar fasciitis in female runners. Clin J Sport Med 2009;19:372-6.

39. Faria A, Gabriel R, Abrantes J, et al. Triceps-surae musculotendinous stiffness: relative differences between obese and non-obese postmenopausal women. Clin Biomech 2009;24:866-71.

40. Wulf M, Wearing SC, Hooper SL, et al. The effect of an in-shoe orthotic heel lift on loading of the achilles tendon during shod walking. J Orthop Sports Phys Ther 2016;46:79-86.

41. Yu J, Wong DW, Zhang $\mathrm{H}$, et al. The influence of high-heeled shoes on strain and tension force of the anterior talofibular ligament and plantar fascia during balanced standing and walking. Med Eng Phys 2016;38:1152-6. 